\title{
La Técnica Grilla de Repertorio (Repertory Grid): Un Método para el Estudio de la Cognición en la Evaluación de Habilidades Docentes
}

\author{
Patricio E. Ramírez(1), Jorge L. Alfaro(1) y Felipe Machorro(2)* \\ Universidad Católica del Norte, (1) Escuela de Ingeniería, (2) Escuela de Ciencias Empresariales, \\ Larrondo 1281, Coquimbo, Chile. (email: patricio.ramirez@ucn.cl; jalfaro@ucn.cl; fmachorro@ucn.cl) \\ * Autor a quien debe ser dirigida la correspondencia
}

Recibido Ago. 5, 2015; Aceptado Oct. 14, 2015; Versión final Nov. 19, 2015, Publicado Abr. 2016

\begin{abstract}
Resumen
El propósito de este estudio es presentar una propuesta metodológica para elicitar los constructos utilizados por los estudiantes para evaluar las habilidades y desempeños docentes. La propuesta se centra en la utilización de la Teoría de Constructos Personales y de su técnica de mapas cognitivos, conocida como Grilla de Repertorio (Repertory Grid). En particular, la propuesta describe las características deseables de los entrevistados, un proceso consistente en cuatro pasos, y el análisis posterior sugerido. Este enfoque se orienta a fortalecer las mayores debilidades de los procesos de evaluación docente: (i) instrumentos adhoc no validados, (ii) basados en teorías que no reflejan la realidad y madurez de un contexto, (iii) conveniencia política y (iv) una baja incidencia y participación percibida por los estudiantes. El estudio muestra que si bien RepGrid fortalece el método de los cuestionarios en el proceso de la evaluación docente, su efectividad final está determinada por su utilización en conjunto con otros métodos de evaluación del desempeño.
\end{abstract}

Palabras clave: grilla de repertorio, evaluación de habilidades docentes, universidad, propuesta

\section{The Repertory Grid Technique: A Method for the Study of Cognition in Teaching Skills Assessment}

\begin{abstract}
The aim of this study is to present a proposal to elicit the constructs used by students to evaluate the abilities and teaching performance. The proposal focuses on the use of personal construct theory and its cognitive mapping technique, known as Repertory Grid. In particular, the proposal describes the desirable characteristics of respondents, a process consisting of four steps, and subsequent analysis suggested. This approach is intended to strengthen major weakness of teaching assessment processes: (i) ad-hoc not validated instruments, (ii) based on theories that do not reflect reality and maturity of a context, (iii) political convenience, and (iv) a low incidence and perceived participation by students. The study shows that, although RepGrid strengthen the questionnaire methodology in the process of teaching evaluation, its final effectivity is determined by its use in conjunction with other evaluation methods.
\end{abstract}




\section{INTRODUCCIÓN}

La evaluación docente es un proceso que se ha vuelto cada vez más importante en las instituciones de educación superior. Esta importancia radica en los dos grandes propósitos para los cuales se lleva a cabo, por una parte, sus resultados son utilizados para contribuir al mejoramiento de la calidad docente (propósito formativo), y por otra parte, son un insumo para la toma de decisiones respecto a la permanencia y promoción de los académicos en las instituciones (propósito sumativo). De esta forma, se ha vuelto un requisito explícito en el sistema de evaluación de las instituciones de educación superior a nivel internacional (Kember, Leung, y Kwan, 2002), lo que conlleva la exigencia constante de mayor objetividad y precisión en su despliegue (Onwuegbuzie, Daniel, y Collins, 2009).

Existen diversos agentes que pueden intervenir en la evaluación de la calidad de la docencia, tales como, los propios docentes, jefes directos, pares académicos y la encuesta de opinión a los estudiantes. Sin embargo, a pesar de la existencia de esta variedad de posibilidades, el instrumento más utilizado es el cuestionario de evaluación aplicado a los estudiantes (Medel y Asún, 2014). En particular, en Chile la importancia de este uso es más notoria debido a la relevancia que tienen en los procesos de acreditación institucional y de programas académicos (Ascencio, 2008).

No obstante de ser una práctica generalizada, y en muchas ocasiones la única fuente de información para tomar decisiones, la aplicación de cuestionarios a los estudiantes para evaluar la calidad docente es un tema que genera polémica. Existen numerosas investigaciones que no han llegado a consenso respecto a su efectividad para mejorar la calidad de los docentes (Sproule, 2000). De hecho, y a pesar de existir una extensa literatura respecto al tópico, aún quedan pendientes aspectos relacionados a la confiabilidad y validez de los instrumentos (Kulik, 2001), posibles sesgos relacionados a aspectos demográficos o institucionales (Arbuckle y Williams, 2003), carencia de marcos adecuados para el diseño de los cuestionarios (Zabaleta, 2007), y diferencias existentes entre los constructos evaluados por los instrumentos y aquellos que los estudiantes consideran deben tener los docentes efectivos (Onwuegbuzie et al., 2007). En este contexto, el tema resulta interesante como objeto de estudio.

Por lo anterior, el propósito de este trabajo es presentar una propuesta metodológica para elicitar los constructos utilizados por los estudiantes para evaluar las habilidades y desempeños docentes. La propuesta se centra en la utilización de la Teoría de Constructos Personales de Kelly (1955/1991) y su técnica de mapas cognitivos conocida como Repertory Grid (RepGrid). Este enfoque tiene como propósito fortalecer las debilidades principales de los procesos de evaluación docente: (i) instrumentos ad-hoc no validados, (ii) basados en teorías que no reflejan la realidad y madurez particular de un entorno, (iii) conveniencia política, y (iv) baja incidencia y participación percibida por los estudiantes (García Garduño, 2014; Giesey, Chen, y Hoshower, 2004; Onwuegbuzie et al., 2007; Richardson, 2005).

\section{EVALUACIÓN DE LAS HABILIDADES DOCENTES}

La evaluación a los docentes por parte de los estudiantes como un instrumento para medir la efectividad de los académicos es una práctica que se ha generalizado en las universidades de todo el mundo (Kuzmanovic, Savic, Popovic, y Martic, 2013; Onwuegbuzie et al., 2009). Estas evaluaciones comenzaron a utilizarse alrededor de los años 20 (Morley, 2013), siendo su propósito original la recolección de información que sirviera para retroalimentar la práctica docente (Sproule, 2000). Durante esa época y hasta los años 60 la participación de los estudiantes era voluntaria; sin embargo, desde 1970, comenzaron a ser de carácter obligatorio y utilizadas para tomar decisiones administrativas (Centra, 1993).

Algunos investigadores argumentan que los cuestionarios son una medida válida de la eficacia de la enseñanza, y que existe suficiente evidencia de cambio por parte de los docentes que sugiere que su desempeño puede mejorar a lo largo del tiempo, además que los sistemas de evaluación tienen un efecto indirecto en los resultados de los estudiantes (Delvaux et al., 2013; Marsh, 1984). Sin embargo, existen algunos resultados empíricos que se contraponen a la idea del mejoramiento docente mediante la evaluación (Bianchini, 2013; Kember et al., 2002), además de poner en duda la validez de la percepción de los estudiantes (Onwuegbuzie et al., 2009) y considerar la evaluación docente como meros concursos de personalidad donde docentes incompetentes son premiados con buenas notas (Centra, 2003; Kulik, 2001).

Otro inconveniente detectado en la evaluación docente se relaciona con el diseño de los instrumentos. A pesar de la extensa investigación realizada, no existe un consenso respecto al marco conceptual en el cual se puedan basar los cuestionarios de evaluación docente (Onwuegbuzie et al., 2009), ni respecto al número y naturaleza de las dimensiones de la docencia que deben ser capturada por los mismos (Jackson et al., 1999). Como resultado, los instrumentos de evaluación docente varían en función de las características y conveniencia de cada institución en particular. Muchas instituciones utilizan instrumentos ad hoc que no han 
sido validados (Richardson, 2005), mientras algunas utilizan instrumentos validados y basados en teorías educacionales. Sin embargo, esto no asegura que se capturen las características deseables de los académicos desde la perspectiva de los estudiantes; ejemplo de esto es el estudio de Onwuegbuzie et al. (2007), en el que identifican nueve características que los estudiantes consideran deben tener los docentes para considerarlos efectivos, las cuales fueron comparadas con el instrumento utilizado para la evaluación docente. Sus resultados indican que tres de las nueve características elicitadas no estaban representadas en ningún ítem del cuestionario de evaluación docente aplicado.

El problema de omitir características deseables en los instrumentos de evaluación docente puede afectar el compromiso de los estudiantes a participar en los procesos mismos de evaluación, ya que su motivación para colaborar en el proceso está asociada al mejoramiento de la enseñanza y de los contenidos del curso (Giesey et al., 2004), en concordancia con lo anterior, Cortés, Campos, y Moreno (2014), reportan en su estudio que la dimensión prioritaria para los estudiantes en la evaluación docente son los Resultados de Aprendizaje. No obstante, según indica García Garduño (2014), en general los alumnos perciben que la evaluación docente tiene fines sumativos, de control y supervisión, por lo que no los consideran útiles ni válidos.

\section{LA TÉCNICA REPGRID}

Según la Teoría de Constructos Personales (Kelly, 1955/1991), las cosas se interpretan por medio de constructos, y en ese proceso le damos sentido a ellas. Este alternativismo constructivo implica que diferentes individuos tienen diferentes maneras de interpretar la misma cosa, como también, que un mismo individuo siempre tiene la opción de interpretar algo en forma diferente en dos ocasiones distintas. En concreto, la realidad está sujeta a muchas construcciones alternativas, y algunas de estas pueden ser más convenientes que otras.

Una forma de reconocer los constructos utilizados por un individuo es el uso de la técnica RepGrid. Un RepGrid de constructos personales es una matriz formada por tres componentes. Primero, las columnas de la matriz representan a los elementos, que especifican una muestra representativa de personas, eventos, situaciones u objetos dentro de un dominio de experiencia particular del individuo. Segundo, las filas de la matriz representan dimensiones bipolares que el individuo emplea para dar sentido a los elementos dispuestos en las columnas; estas dimensiones bipolares constituyen los constructos personales, los cuales son elicitados al considerar similitudes y diferencias entre los elementos. Y tercero, cada intersección entre fila y columna señala la puntuación/relación de un elemento en/con un constructo personal (Jankowicz, 2005). La tabla 1 muestra un ejemplo de RepGrid de tres elementos (A, B, C) y tres constructos (X, Y, Z).

Tabla 1: RepGrid de tres elementos

\begin{tabular}{|l|l|l|l|}
\hline & Elemento $A$ & Elemento $B$ & Elemento $C$ \\
\hline Constructo $X$ & Relación $A, X$ & Relación $B, X$ & Relación $C, X$ \\
\hline Constructo $Y$ & Relación $A, Y$ & Relación $B, Y$ & Relación $C, Y$ \\
\hline Constructo $Z$ & Relación $A, Z$ & Relación $B, Z$ & Relación $C, Z$ \\
\hline
\end{tabular}

Por otra parte, en la medida en que individuos tienen experiencias similares a otros individuos dentro de una comunidad, sus constructos personales tienden a ser similares a los de dicha comunidad, es decir, comparten una visión o interpretación de los eventos (corolario de comunalidad) (Kelly, 1955/1991). Por tanto, es posible reconocer la visión compartida de una comunidad (en relación a elementos de un dominio de experiencia determinado) a través del uso sistemático de la técnica RepGrid en individuos pertenecientes a ella (Tan y Hunter, 2002). En particular, este enfoque admite que es posible entender la cognición organizacional midiendo y entendiendo la cognición individual (Weick, 2012).

Existen importantes aspectos a considerar en el diseño de un RepGrid de constructos personales asociados a cada uno de sus componentes. La selección de los elementos, dentro de un dominio específico de experiencia para el individuo al cual se le aplicará el instrumento, es fundamental para el diseño de la técnica. En este punto existen dos alternativas, por una parte, es posible que los elementos sean entregados por el investigador, o por otra, que sea el individuo participante en la investigación quien los provea. Si bien la primera alternativa entrega una mayor homogeneidad para el análisis, como la posibilidad de que sea guiada por la teoría o por el foco del investigador, la segunda alternativa, asegura que los elementos sean relevantes para los participantes en la investigación (Reger, 1990). En cualquier alternativa, la reglas indican que los elementos seleccionados deben ser discretos, homogéneos, no evaluativos y representativos del área a 
investigar (Tan y Hunter, 2002). La elicitación de constructos es otro aspecto importante a considerar en el diseño de la técnica. Si bien es posible que el investigador provea los constructos a ser evaluados, la forma clásica es la elicitación a través de triadas a azar. En este método, luego se seleccionar tres elementos al azar de entre todos los elementos del RepGrid, se le pide al entrevistado identificar de alguna manera dos elementos que son semejantes entre sí pero diferentes de un tercero. Finalmente, para la puntación/relación entre elementos y constructos existen tres métodos: 1) dicotómico, que solicita al participante colocar a cada elemento a un lado, $u$ al otro, de los polos del constructo; 2) ranking, que solicita al participante poner en orden los elementos entre dos polos; y 3) calificación, que pide al participante poner una calificación en una escala de puntos (Tan y Hunter, 2002).

\section{REPGRID EN EDUCACION SUPERIOR}

El uso de RepGrid en el ámbito de la educación superior ha sido variado y más bien reducido en el tiempo, en comparación a su empleo en disciplinas como la psicología. De hecho, según el estudio de Saúl y sus colegas (Saúl et al., 2012) sobre el uso de la técnica entre los años 1998-2007, de un total de 468 artículos, 262 fueron clasificados en el área de la psicología y los otros 206 se distribuyeron en otras 17 disciplinas. De éste último total, sólo el 1,5\% se clasificó en el ámbito de la educación

Por otra parte, los esfuerzos científicos que utilizan RepGrid en educación superior se han apoyado generalmente con otras técnicas de investigación, tanto cualitativas como cuantitativas. En 1992 sobresale por su citación el trabajo de Solas (1992), quien observa la oportunidad de potenciar el entendimiento del proceso de enseñanza y aprendizaje extendiendo una invitación al diálogo de profesores y estudiantes. Acentúa el uso de la autobiografía y RepGrid de forma de mantener, a la vez de revelar, las perspectivas particulares de estudiantes y profesores. En Pill (2005) se reporta una investigación que expone como propósito identificar y evaluar modelos de desarrollo profesional empleados en la educación y práctica de profesores novicios en nueve instituciones de educación superior. Este estudio cualitativo utilizó RepGrid y entrevistas semi-estructuradas y sus resultados reportan la elicitación de cuatro constructos, dos de los cuales son identificados por la técnica RepGrid y no por las entrevistas. Kreber (2005) expone un estudio exploratorio de la reflexión en el proceso de enseñanza en educación superior. Treinta y seis profesores en ciencias participaron en una entrevista semi-estructurada empleando RepGrid. El estudio identificó, entre otros resultados, indicadores concretos de reflexión, los cuales pueden ser de utilidad en la evaluación del cuerpo académico.

Guo et al. (2011) desarrollan una investigación que busca determinar factores que motivan a los estudiantes a emplear medios de comunicación basados en tecnología de información en el contexto del proceso de aprendizaje. Se empleó RepGrid para realizar entrevistas a 17 estudiantes, con las cuales se identificaron ocho categorías de razones de los estudiantes para el uso de los medios de comunicación en contextos de aprendizaje. Ribeiro et al. (2012) exponen los resultados de un estudio en el cual se utilizó el RepGrid para evaluar los niveles de síntomas psicológicos, habilidades de resolución de problemas y auto-construcciones en 28 estudiantes, tanto al inicio como al término del primer año de universidad. Se obtuvieron 15 constructos que fueron utilizados en un estudio cuantitativo posterior. En 2013, Carolin Kreber y Monika Klampfleitner publican un estudio respecto del significado e incidencia de la autenticidad en la enseñanza (Kreber y Klampfleitner, 2013). Esta investigación emplea la técnica RepGrid y grupos focales para determinar y contrastar las concepciones de autenticidad de nueve profesores y 46 estudiantes. En este caso se realiza tanto un análisis cuantitativo como cualitativo de los resultados obtenidos por el uso de RepGrid para encontrar las diferencias de percepción entre la muestra de estudiantes respecto a los profesores. Finalmente, Stevens et al. (2014), empleando los enfoques de gratificación y usos, y aprendizaje mediado por tecnología, desarrollan una investigación de las motivaciones de estudiantes universitarios para emplear tecnologías Web 2.0 en su proceso de aprendizaje. Se emplea Reportory Grid, 16 entrevistados, para elicitar 11 motivaciones de los estudiantes para usar tecnología.

Puede observarse que en el campo de la educación, la técnica RepGrid ha sido utilizada y validada como una forma eficaz para abordar investigaciones en las cuales es necesario elicitar constructos personales; sin embargo, se aprecia una ausencia de la misma en el ámbito de la evaluación docente, razón por la cual, en la siguiente sección se plantea un procedimiento para su utilización en dicho ámbito.

\section{PROPUESTA METODOLÓGICA}

Considerando lo expuesto anteriormente, se propone el siguiente método para la elicitación de los constructos utilizados por los estudiantes universitarios en la evaluación de las habilidades docentes. 


\section{Sobre los entrevistados.}

Los entrevistados deben ser alumnos universitarios con experiencias similares. En particular, se propone que sean alumnos de último año, de disciplinas afines (por ejemplo, de carreras de ingeniería), y dentro de un área geográfica particular (por ejemplo, de una misma sede del centro de estudios). El número total de entrevistados debe estar en el rango de 15 a 25, en concreto, se debe parar de realizar entrevistas cuando ya no emerjan nuevos constructos (Tan y Hunter, 2002).

\section{Sobre el proceso.}

Siguiendo a Hunter (1997) se ha diseñado el siguiente esquema de cuatro pasos para la entrevista: introducción a la entrevista, elementos del repgrid, elicitación de los constructos a través de triadas, y revisión

Paso 1: Introducción a la entrevista. 1.a. Consultar al entrevistado sobre su experiencia como alumno de profesores universitarios, y luego introducir la evaluación de habilidades docentes de estos profesores como tema de la entrevista. 1.b. Explicar en forma general el proceso de la entrevista. 1.c. Firma de acuerdo de confidencialidad y consentimiento.

Paso 2: Elementos del RepGrid. 2.a. Solicitar al entrevistado que identifique 6 profesores universitarios (elementos) con los que ha interactuado como alumno regular en algún curso dictado por ellos. Cada elemento es escrito en una tarjeta distinta. 2.b. Adicionalmente se integran dos tarjetas a modo de anclaje, una con el profesor 'ideal', y otra con el profesor 'incompetente'. En total se obtienen 8 tarjetas.

Paso 3: Elicitación de los constructos a través de triadas. 3.a. Se pide al entrevistado seleccionar 3 tarjetas al azar, entre el total de 8 , y luego se le realiza en siguiente cuestionamiento: En relación a las habilidades docentes de los profesores, ¿En qué aspecto se parecen dos de estos profesores y son a la vez distintos del tercero?, y a continuación: ¿En qué sentido se diferencia el tercer profesor de los otros dos? 3.b. Se debe registrar las notas detalladas de la entrevista. 3.c. Se le pide al entrevistado calificar, en relación al constructo elicitado, a cada elemento en una escala de 1 (el final positivo del constructo) a 7 (el final negativo del constructo). El Paso 3 se repite hasta que el entrevistado no pueda encontrar nuevos constructos.

Paso 4. Revisión. 4.a. Se revisan los resultados obtenidos con el objeto de encontrar constructos o elementos que sean muy similares o muy diferentes. Este análisis se comenta con el entrevistado. 4.b. Se solicitan más comentarios al entrevistado en relación al análisis, en particular, sobre si la RepGrid registra en forma justa lo que ha declarado durante la entrevista. 4.c. Se realiza el cierre de la entrevista consultando si existen preguntas o inquietudes sobre el proceso en general.

\section{Sobre el análisis posterior.}

El primer lugar, se propone un análisis de los constructos que permita obtener agrupaciones significativas de categorías de constructos. Varias son las técnicas posibles en esta etapa: recuento de frecuencias, análisis de contenido, focalización visual y análisis de conglomerados (Napier, Keil, y Tan, 2009). Posteriormente, siguiendo a Preiss (2000), se propone realizar un ranking de los constructos a través del Proceso Analítico Jerárquico. Para ello se debe considerar la opinión de todos los alumnos entrevistados.

\section{PRUEBA PILOTO}

Con el fin de evaluar el método propuesto se realizó un piloto a 15 estudiantes de ingeniería, 8 mujeres y 7 hombres. Todos estos estudiantes eran de últimos años de carreras de ingeniería.

Luego de realizar las entrevistas y a partir del análisis de los resultados se pudieron elicitar las siguientes habilidades docentes:

1. Sabe lo que enseña.

2. Es empático.

3. Da ejemplos de la vida real.

Más allá de estos resultados iniciales, debemos destacar algunos puntos del método. Primero, el método propuesto fue eficiente, pues logró en poco tiempo alcanzar con cada entrevistado un conjunto de habilidades que ellos consideraban importantes al momento de evaluar a un profesor, en promedio las entrevistas fueron de 35 minutos. Segundo, mayoritariamente los entrevistados se mostraron muy conformes con la forma explícita en que fue reflejado su forma de valorizar a un docente en la grilla. Tercero, el hecho que los 
elementos de la grilla (docentes) no fueron registrados por los investigadores, otorgó un alto grado de sinceridad de los entrevistados lo que causa una mayor fiabilidad de las habilidades elicitadas. Y por último, el hecho de realizar una revisión de los resultados con cada entrevistado mostró la posible causalidad o correlación de algunas habilidades docente, este punto deberá ser evaluado en futuras aplicaciones del método.

\section{CONCLUSIONES}

A modo de conclusiones de este trabajo se pueden indicar cinco puntos. Primero, la evaluación de habilidades docentes es un tema crucial en la educación superior, sin embargo se detecta una carencia en la aproximación científica al fenómeno, produciéndose con ello un abierto debate sobre la real utilidad de estos mecanismos de control. Este enfoque tiene como propósito fortalecer las debilidades principales de los procesos de evaluación docente: (i) instrumentos ad-hoc no validados, (ii) basados en teorías que no reflejan la realidad y madurez particular de un entorno, (iii) conveniencia política y (iv) baja incidencia y participación percibida por los estudiantes. Segundo, la técnica RepGrid ha sido utilizada en variados ámbitos de las ciencias sociales, sin embargo, en el ámbito de la educación se aprecia un uso limitado de ella. Los resultados de este uso nos revelan tanto su potencial en cuanto a técnica, como la riqueza de los análisis posteriores a su aplicación. Tercero, este trabajo potencia la evaluación docente basada en cuestionarios, dado que a partir de los constructos elicitados será factible especificar instrumentos de evaluación docente, pero reconociendo que los estudiantes usan sus propios constructos para entender e interpretar eventos que suceden a su alrededor y que estos constructos están moderados por sus experiencias individuales. Cuarto, si bien RepGrid fortalece el método de los cuestionarios en el proceso de la evaluación docente, su efectividad final está determinada por su utilización en conjunto con otros métodos de evaluación del desempeño (Risquez, Vaughan, y Murphy, 2015). Por ejemplo, evaluación de pares y portafolios de evidencias (Alderman, Towers, y Bannah, 2012). Quinto y último, en este trabajo fue posible desarrollar una propuesta metodológica en base a la técnica RepGrid para entender cómo son evaluadas las habilidades docentes en educación superior por parte de los alumnos, la cual fue aplicada en una prueba piloto que confirma su eficiencia y pertinencia.

\section{REFERENCIAS}

Alderman, L., Towers, S., y Bannah, S., Student feedback systems in higher education: a focused literature review and environmental scan, doi: 10.1080/13538322.2012.730714. Quality in Higher Education, 18(3), 261$280(2012)$

Arbuckle, J., y Williams, B., Students' Perceptions of Expressiveness: Age and Gender Effects on Teacher Evaluations, doi: 10.1023/A:1025832707002. Sex Roles, 49(9-10), 507-516 (2003)

Ascencio, J. S., Diagnóstico preliminar sobre evaluación de la docencia universitaria. Una aproximación a la realidad en las universidades públicas y/o estatales de Chile. Revista Iberoamericana de Evaluación Educativa, 1(3), 67-84 (2008)

Bianchini, S., Feedback effects of teaching quality assessment: macro and micro evidence, doi: 10.1080/02602938.2013.842957. Assessment \& Evaluation in Higher Education, 39(3), 380-394 (2013)

Centra, J. A., Reflective Faculty Evaluation: Enhancing Teaching and Determining Faculty Effectiveness. The Jossey-Bass Higher and Adult Education Series, ERIC, San Francisco CA (1993)

Centra, J. A., Will Teachers Receive Higher Student Evaluations by Giving Higher Grades and Less Course Work?, doi: 10.1023/A:1025492407752. Research in Higher Education, 44(5), 495-518 (2003)

Cortés, E., Campos, M., y Moreno, M. P., Priorización de las dimensiones de Evaluación al Desempeño Docente por el estudiante, en tres áreas del conocimiento, doi: 10.4067/S0718-50062014000200002. Formación Universitaria, 7, 3-10 (2014)

Delvaux, E. y otros cinco autores, How may teacher evaluation have an impact on professional development? A multilevel analysis, doi: 10.1016/j.tate.2013.06.011. Teaching and Teacher Education, 36(0), 1-11 (2013)

García Garduño, J. M., ¿Para qué sirve la evaluación de la docencia? Un estudio exploratorio de las creencias de los estudiantes. Archivos Analíticos de Políticas Educativas, 22(15), 1-24 (2014)

Giesey, J. J., Chen, Y., y Hoshower, L. B., Motivation of Engineering Students to Participate in Teaching Evaluations, doi: 10.1002/j.2168-9830.2004.tb00819.x. Journal of Engineering Education, 93(4), 303-312 (2004) 
Guo, Z. X., Lu, X. W., Li, Y., y Li, Y. F., A Framework of Students' Reasons for Using CMC Media in Learning Contexts: A Structural Approach, doi: 10.1002/asi.21631. Journal of the American Society for Information Science and Technology, 62(11), 2182-2200 (2011)

Hunter, M. G., The use of RepGrids to gather interview data about information systems analysts. Information systems journal, 7(1), 67-81 (1997)

Jackson, D. L. y otros cinco autores, The dimensions of students' perceptions of teaching effectiveness. Educational and Psychological Measurement, 59(4), 580-596 (1999)

Jankowicz, D., The easy guide to repertory grids, John Wiley \& Sons, Chichester (2005)

Kelly, G., Psychology of Personal Constructs (Vols. 1 and 2), Routledge, London (1955/1991)

Kember, D., Leung, D. Y. P., y Kwan, K. P., Does the Use of Student Feedback Questionnaires Improve the Overall Quality of Teaching?, doi: 10.1080/0260293022000009294. Assessment \& Evaluation in Higher Education, 27(5), 411-425 (2002)

Kreber, C., Reflection on teaching and the scholarship of teaching: Focus on science instructors, doi: 10.1007/s10734-004-6360-2. Higher Education, 50(2), 323-359 (2005)

Kreber, C., y Klampfleitner, M., Lecturers' and students' conceptions of authenticity in teaching and actual teacher actions and attributes students perceive as helpful, doi: 10.1007/s10734-013-9616-x. Higher Education, 66(4), 463-487 (2013)

Kulik, J. A., Student Ratings: Validity, Utility, and Controversy, doi: 10.1002/ir.1. New Directions for Institutional Research, 2001(109), 9-25 (2001)

Kuzmanovic, M., Savic, G., Popovic, M., y Martic, M., A new approach to evaluation of university teaching considering heterogeneity of students' preferences, doi: 10.1007/s10734-012-9596-2. Higher Education, 66(2), 153-171 (2013)

Marsh, H. W., Students' evaluations of university teaching: Dimensionality, reliability, validity, potential baises, and utility. Journal of Educational Psychology, 76(5), 707 (1984)

Medel, R., y Asún, R., Encuestas de evaluación docente y sesgos de género: un estudio exploratorio. Calidad en la educación, 171-199 (2014)

Morley, D., Assessing the reliability of student evaluations of teaching: choosing the right coefficient, doi: 10.1080/02602938.2013.796508. Assessment \& Evaluation in Higher Education, 39(2), 127-139 (2013)

Napier, N. P., Keil, M., y Tan, F. B., IT project managers' construction of successful project management practice: a repertory grid investigation, doi: 10.1111/j.1365-2575.2007.00264.x. Information systems journal, 19(3), 255-282 (2009)

Onwuegbuzie, A. J., Daniel, L. G., y Collins, K. M. T., A meta-validation model for assessing the score-validity of student teaching evaluations, doi: 10.1007/s11135-007-9112-4. Quality \& Quantity, 43(2), 197-209 (2009)

Onwuegbuzie, A. J. y otros cinco autores, Students' Perceptions of Characteristics of Effective College Teachers: A Validity Study of a Teaching Evaluation Form Using a Mixed-Methods Analysis, doi: 10.3102/0002831206298169. American Educational Research Journal, 44(1), 113-160 (2007)

Pill, A., Models of professional development in the education and practice of new teachers in higher education. Teaching in Higher Education, 10(2), 175-188 (2005)

Preiss, K. J., A two-stage process for eliciting and prioritising critical knowledge. Journal of Knowledge Management, 4(4), 328-336 (2000)

Reger, R. K., The repertory grid technique for eliciting the content and structure of cognitive constructive systems, en Mapping Strategic Thought por A. S. Huff, (pp. 301-309), John Wiley \& Sons, New York (1990)

Ribeiro, E., Feixas, G., Maia, A., Senra, J., y Dada, G., Changes in the Construct Systems of First-Year University Students: Impact on Psychological Symptoms and Problem-Solving Skills, doi: 10.1080/10720537.2012.629119. Journal of Constructivist Psychology, 25(2), 170-180 (2012) 
Richardson, J. T., Instruments for obtaining student feedback: A review of the literature. Assessment \& Evaluation in Higher Education, 30(4), 387-415 (2005)

Risquez, A., Vaughan, E., y Murphy, M., Online student evaluations of teaching: what are we sacrificing for the affordances of technology?, doi: 10.1080/02602938.2014.890695. Assessment \& Evaluation in Higher Education, 40(1), 120-134 (2015)

Saúl, L. A. y otros cinco autores, Bibliometric review of the repertory grid technique: 1998-2007. Journal of Constructivist Psychology, 25(2), 112-131 (2012)

Solas, J., Investigating Teacher and Student Thinking about the Process of Teaching and Learning Using Autobiography and Repertory Grid, doi: 10.3102/00346543062002205. Review of Educational Research, 62(2), 205-225 (1992)

Sproule, R., Student Evaluation of Teaching: A Methodological Critique education policy analysis archives, 8, $50(2000)$

Stevens, K., Guo, Z., y Li, Y., Understanding Technology Mediated Learning in Higher Education: A Repertory Grid Approach, Thirty Fifth International Conference on Information Systems, 1-20, Auckland 14 a 17 de diciembre (2014)

Tan, F. B., y Hunter, M. G., The repertory grid technique: A method for the study of cognition in information systems. Mis Quarterly, 26(1), 39-57 (2002)

Weick, K. E., Making Sense of the Organization, John Wiley \& Sons, West Sussex (2012)

Zabaleta, F., The use and misuse of student evaluations of teaching, doi: 10.1080/13562510601102131. Teaching in Higher Education, 12(1), 55-76 (2007) 